\title{
Prix Des Matieres Premieres D'exportation Et Taux De Change Effectifs Reels Dans Les Pays De L'uemoa
}

\author{
Hermann Abbe \\ Doctor in Economics, International Economic Option, \\ Teacher and Researcher attached to the Center for Research \\ and Action for Peace (CERAP Ivory Coast), University Pole \\ Hervé Daniel Gbame \\ Doctor in Economics, Industrial Economics Option, \\ Teacher and Researcher at the University Institute of Abidjan (Ivory Coast)
}

\section{Résumé}

Cet article examine les effets de la fluctuation des prix des matières premières d'exportations sur les taux de change effectif dans l'UEMOA sur la période 1999 - 2016 afin d'en apprécier le comportement de consommation des ménages. A cet égard, l'approche PMG (Pooling Mean Group) sur données de panel appliquée à un modèle BEER a été utilisée. Les résultats montrent que les prix des matières premières sont le principal facteur d'appréciation réelle du taux de change à long terme dans l'UEMOA. Cependant, cette variable n'aide pas à expliquer la dynamique de court terme. Par ailleurs, nous montrons que la monnaie d'ancrage est un facteur d'appréciation à court terme et non à long terme. Cela justifie les perpétuelles rumeurs de dévaluation suite aux surévaluations subites de l'euro. Ces résultats suggèrent des mesures préventives pour contenir les effets pervers des éventuelles fluctuations brusques de la monnaie d'ancrage sur les consommations des ménages.

Mots clés : Taux de change effectif réel, Termes de change des matières premières, l'UEMOA, dynamique à court et long terme. 


\title{
Commodity Prices Exporting and Real Effective Exchange Rates in Waemu Countries
}

\author{
Hermann Abbe \\ Doctor in Economics, International Economic Option, \\ Teacher and Researcher attached to the Center for Research \\ and Action for Peace (CERAP Ivory Coast), University Pole \\ Hervé Daniel Gbame \\ Doctor in Economics, Industrial Economics Option, \\ Teacher and Researcher at the University Institute of Abidjan (Ivory Coast)
}

\begin{abstract}
This article examines the effects of the fluctuation of the prices of exports commodities on the effective exchange rate in WAEMU over the period 1999 - 2016 in order to appreciate the consumption behaviour of households. In this respect, the Pooled Mean Group (PMG) approach applied to a BEER model was used. The results show that commodity prices are the main factor in the real appreciation of the long-term exchange rate in WAEMU. However, this variable does not help to explain the short-term dynamics. Moreover, we show that the anchor currency is a factor of appreciation in the short term and not in the long term. This justifies the perpetual rumours of devaluation following the sudden overvaluation of the euro. These results suggest preventive measures to contain the adverse effects of possible abrupt fluctuations of the anchor currency on household consumption.
\end{abstract}

Keywords: Real effective exchange rate, commodity exchange terms, UEMOA, short and long term dynamics.

\section{Introduction}

Théoriquement, la problématique de la relation entre les termes de l'échange des matières premières et le taux de change effectif réel date des années 1980 (Neary, 1988). Elle est assez récente. Par ailleurs, elle a occupé une place importante dans la littérature empirique au début des années 2000 suite aux travaux de Chen et Rogoff (2003) et Cashin et al, (2004).

Chen et Rogoff (2003) ont, en effet, trouvé une relation de cointégration entre ces deux variables pour trois économies développés (Australie, Canada et Nouvelle-Zélande) selon laquelle les produits primaires 
constituent une part importante de leurs exportations. Cashin et al, (2004) retrouvent également ces mêmes résultats de cointégration entre les termes de l'échange et taux de change effectif réel. Leurs travaux réalisés à partir des données du FMI sur les prix mondiaux de 44 produits de base et des parts nationales des exportations de produits de base appliquées à 58 pays ont confirmé l'existence d'une relation à long terme entre le taux de change réel national et les prix réels des produits de base dans environ un tiers des pays exportateurs de produits de base.

Malgré la pertinence des résultats de ces auteurs qui ont mis en évidence un lien significatif et positif entre ces deux variables, qualifiant les monnaies de certains pays exportateurs de produits de base de «devisesmatières premières», les études empiriques consacrées aux pays en développement sont rares. Or ces pays fournissent un vaste champ d'investigations en raison de leur spécialisation dans la production des matières premières. Au nombre des rares études ayant abordé la question, on note celle de Coudert et al. (2010). Ces auteurs montrent que les taux de change réels évoluent à long terme avec les prix des matières premières agricoles et énergétiques de 52 pays en développement (dont certains pays de la zone CFA). Toutefois, avec un groupe de pays hétérogènes, les études ne tiennent pas compte de l'arrangement monétaire et de change des pays. En effet, la mise en commun de pays ayant des propriétés économiques et géographiques différentes conduit très souvent à des résultats trompeurs. Par ailleurs, il apparait que cette poignée d'études se concentrent sur les pays d'Amérique latine et d'Asie ou les pays producteurs de produits énergétiques (notamment pétroliers et miniers), comme on peut l'apercevoir dans le tableau 1 (voir annexe).

Cette étude a pour objectif de répondre en partie à ces insuffisances en se concentrant sur les pays de l'UEMOA qui, spécialisés dans l'exportation de matières premières non transformées, partagent une monnaie commune (le F CFA). Elle cherche donc à évaluer les effets des fluctuations des prix de matières premières sur les taux de change effectif réels dans l'UEMOA sur la période de 1999 à 2016. Eu égard aux travaux antérieurs (Cashin et al, 2004 et Coudert et al, 2010), ce travail constitue, à notre connaissance, la première application dans l'UEMOA. Pour ce faire, il essaie de répondre à deux principales questions : Existe-il une relation de long terme entre les prix des matières premières et les taux de change effectifs réels des pays de l'UEMOA? Quelles sont les modalités de convergence des taux de change effectifs réels vers leurs niveaux d'équilibre dans l'UEMOA après un déséquilibre consécutif à une chute des prix des matières premières?

En outre, les résultats de cette étude pourront instruire les décideurs de la zone sur les comportements des ménages suite à une chute (hausse) des prix de ces matières. 
Suivant le modèle théorique (Cashin et al, 2004) qui servira de guide à notre recherche empirique, nous montrerons qu'une augmentation du prix international des matières premières a pour conséquence d'augmenter les salaires dans le secteur des produits de base. Sous l'hypothèse d'une mobilité de la main d'œuvre, cette hausse des revenus se généralise aux autres secteurs d'activité, ce qui devrait engendrer une appréciation du taux de change réel. En revanche, on apprend aisément de cette même hypothèse l'ampleur, les coûts économiques et sociaux que génèrent les baisses récurrentes des prix mondiaux des matières premières dans l'UEMOA. En effet, selon la banque mondiale (2005), en raison de l'effondrement des cours des matières premières entre juin 2014 et décembre 2015, l'activité économique a ralenti en Afrique subsaharienne avec en moyenne une croissance du PIB de l'ordre de 3,0\%, au lieu des 4,5\% enregistrés en 2014. Il s'agit du taux de croissance le moins élevé depuis 2009. C'est pourquoi, établir un diagnostic clair et précis du lien entre les prix des matières premières et les taux de change effectif réels dans l'UEMOA s'avère important. Cette préoccupation trouve toute sa raison dans le fait que le taux de change effectif réel est à la fois, une variable économique clé qui permet d'évaluer la compétitivité-prix d'un pays et un enjeu crucial dans les économies où les revenus proviennent principalement des exportations.

La suite de cet article est structurée de la façon suivante : la section 2 fournit la méthodologie de recherche en exposant le cadre théorique et empirique du lien entre les prix de matières premières et les taux de change réels. La section 3 présente l'estimation de la relation de long terme entre le taux de change réel et ses fondamentaux. Enfin, la quatrième section fait une synthèse et conclut l'article.

\section{Méthodologie et données}

Cette section se concentre sur les cadres théorique et empirique de la relation entre les fluctuations des prix des matières premières et le taux de change réel dans la zone UEMOA.

\subsection{Le modèle théorique}

Théoriquement, les études sur le lien entre les prix des matières premières et le taux de change réel s'inscrivent dans les travaux pionniers de Neary [1988], De Gregorio et Wolf [1994], Chen et Rogoff [2003], Cashin et al. [2004]. Dans ces travaux, les auteurs considèrent un modèle à deux secteurs : l'un représentant les biens échangeables ou les matières premières dont les prix se déterminent sur un marché mondial et l'autre, les biens non échangeables.

Suivant Cashin et al. [2004]), notre modèle théorique comprend quatre parties. La première présente l'activité de production domestique. La 
deuxième partie décrit le comportement des consommateurs locaux. La troisième analyse les activités de production et consommation extérieures. La dernière partie est réservée au lien entre les prix des matières premières et le taux de change réel.

\section{L'activité de production locale}

On suppose que l'économie nationale est composée de deux secteurs différents : l'un produisant un bien qui est entièrement exporté, appelé « matière première », et l'autre produisant un bien non commercialisé. Une hypothèse simplificatrice de ce modèle suppose que la production de ces deux types de biens est basée sur un seul facteur de production à savoir la maind'œuvre. Par conséquent, les fonctions de production du produit d'exportation $\left(y_{x}\right)$ et du bien non échangeable $\left(y_{N}\right)$ se présentent comme suit :

$$
\begin{gathered}
y_{x}=a_{x} L_{x} \\
y_{N}=a_{N} L_{N}
\end{gathered}
$$

Où $L_{x}, L_{N}, a_{x}$ et $a_{N}$ sont respectivement la quantité de maind'œuvre allouée au secteur de la matière première, l'allocation de maind'œuvre dans le secteur non échangeable, la productivité du travail dans le secteur des biens d'exportations et la productivité du travail dans la production du bien non échangeable

Sous l'hypothèse d'une mobilité du travail, les salaires (w) restent les mêmes dans les deux secteurs. Alors, la maximisation du profit dans les deux secteurs donne :

$$
\begin{array}{r}
P_{x}=\frac{w}{a_{x}} \\
P_{N}=\frac{w}{a_{N}}
\end{array}
$$

Où $P_{x}$ et $P_{N}$ représentent les prix des biens échangeables et des biens non échangeables. En équilibre, la productivité marginale du travail doit être égale au salaire réel dans chaque secteur. Nous supposons que le prix du produit d'exportation est exogène pour les entreprises (compétitives) dans le secteur des matières premières et qu'il y a une concurrence parfaite dans le secteur non commercialisé. Par conséquent, nous pouvons réécrire le prix du bien non échangeable afin de l'exprimer en fonction du prix du produit d'exportation et des productivités relatives entre les secteurs d'exportation et non échangeable. Ainsi, on a :

$$
P_{N}=\frac{a_{x}}{a_{N}} P_{x}
$$


Il apparait que le prix relatif du bien non échangeable $\left(P_{N}\right)$ par rapport à celui de la matière première $\left(P_{x}\right)$ est entièrement déterminé par des facteurs technologiques et est indépendant des conditions de la demande. De plus, une hausse du prix de la matière première provoque une augmentation des salaires dans ce secteur. Sous l'hypothèse de la mobilité du travail, la hausse peut s'étendre au secteur du bien non échangeable, conduisant à un accroissement du niveau général des prix dans le pays considéré.

\section{Le comportement des ménages}

On suppose que les ménages de l'économie considérée fournissent un travail inélastique dont la dotation totale est $L=L_{x}+L_{N}$ et consomment un bien non-échangeable et un bien échangeable. Notons que le bien échangeable est d'origine étrangère et la matière première produite localement est entièrement exportée. Ainsi, chaque individu choisit la consommation des biens non échangeable et échangeable pour maximiser son utilité, ce qui suppose une augmentation du niveau de la consommation globale $(C)$ de sorte que :

$$
C=k C_{N}^{\gamma} C_{T}^{1-\gamma}
$$

Où $C_{N}$ représente la consommation du bien non échangeable, $C_{T}$ la consommation du bien importé et $k=1 /\left[\gamma^{\gamma}(1-\gamma)^{(1-\gamma)}\right]$ est une constante.

Par ailleurs, le prix minimum d'une unité de consommation de $\mathrm{C}$ est donné par:

$$
P=P_{N}^{\gamma} P_{T}^{1-\gamma}
$$

Où $P_{T}$ est le prix en monnaie locale d'une unité du bien échangeable. $P$ est défini comme l'indice des prix à la consommation. Par ailleurs, le prix du bien échangeable peut être exprimé en fonction de celui des biens importés comme suit :

$$
P_{T}=\frac{P_{T}^{*}}{E}
$$

Où $E$ est le taux de change nominal défini au certain c'est-à-dire le nombre de la monnaie étrangère par unité de la monnaie locale, et $P_{T}^{*}$ est le prix du bien échangeable (importé) en termes de devises. Nous spécifions maintenant plus en détail le reste du monde.

\section{La production et la consommation extérieures}

On suppose que l'extérieur produit trois types de biens : un bien non échangeable qui est consommé par les étrangers, un bien intermédiaire et un bien final. 
Sous l'hypothèse d'un seul facteur, le facteur travail, la technologie disponible pour la production du bien non échangeable se présente comme suit : $y_{N}^{*}=a_{N}^{*} L_{N}^{*}$.

On note que le bien intermédiaire est utilisé dans la production du bien final. De même, la fonction de production dont disposent les entreprises de ce secteur est donnée par : $y_{I}^{*}=a_{I}^{*} L_{I}^{*}$

Par ailleurs, nous admettons que la mobilité de la main-d'œuvre dans les secteurs (étrangers) garantit que le salaire (étranger) est réparti entre les secteurs. Ainsi, nous exprimons le prix du bien non échangé étranger en fonction des productivités relatives et du prix du bien intermédiaire comme suit:

$$
P_{N}^{*}=\frac{a_{I}}{a_{N}} P_{I}^{*}
$$

Quant à la production du bien final, sa technologie utilise deux intrants à savoir une matière première produite par plusieurs pays, notamment notre économie domestique $\left(y_{I}\right)$ et le bien intermédiaire étranger $\left(y_{X}\right)$ dont la fonction de production du bien final est : $Y_{T}^{*}=v\left(Y_{I}^{*}\right)^{\beta}\left(Y_{X}^{*}\right)^{1-\beta}$. Il ressort de cette fonction que le coût unitaire du bien échangeable en monnaie étrangère est donné par: $P_{T}^{*}=\left(P_{I}^{*}\right)^{\beta}\left(P_{X}^{*}\right)^{1-\beta}$. Admettant que les ménages étrangers consomment le bien final et le bien non échangeable, fournissent par la même occasion une force de travail inélastique aux trois différents secteurs. Ainsi, l'indice à la consommation étranger est :

$$
P^{*}=\left(P_{N}^{*}\right)^{\gamma}\left(P_{T}^{*}\right)^{1-\gamma}
$$

\section{La détermination du taux de change réel}

Le taux de change réel dans l'économie domestique est défini par le rapport entre le prix de consommation intérieure et le prix étranger de consommation $\left(E P / P^{*}\right)$. En combinant, les équations (5), (7), (9) et (10), nous pouvons montrer que:

$$
\frac{E P}{P^{*}}=\left(\frac{a_{X}}{a_{I}} \frac{a_{N}^{*}}{a_{N}} \frac{P_{X}^{*}}{P_{I}^{*}}\right)^{\gamma}
$$

Où $\left(\frac{P_{X}^{*}}{P_{I}^{*}}\right)$ est le prix de la matière première par rapport au bien étranger intermédiaire déterminé par les prix étrangers, $\left(\frac{a_{X}}{a_{I}}\right)$ représente les écarts de productivité entre les secteurs de l'exportation et de l'importation 
étrangère et $\left(\frac{a_{N}^{*}}{a_{N}}\right)$ désigne les écarts de productivité entre les secteurs des biens non échangeables locaux et étrangers. On retrouve au travers de ces deux derniers termes un mécanisme proche de l'effet Balassa-Samuelson, selon lequel les gains de productivité réalisés dans le secteur des biens échangeables déclenchent une hausse des salaires qui s'étend à l'ensemble de l'économie et conduit à une appréciation du taux de change réel.

\subsection{Modèle empirique}

La méthodologie d'estimation de la relation entre le taux de change effectif réel et les prix de matières dans la zone UEMOA est l'approche BEER (Behavioral Equilibrium Exchange Rate) de Clark et MacDonald [1998]) d'une part et s'inspire des travaux de Cashin et al. [2004], notamment le modèle théorique présenté précédemment, et des études de Coudert et al. [2010] qui ont travaillé sur les pays en développement intégrant les pays de la zone UEMOA, d'autre part. Elle consiste fondamentalement à estimer une relation à long terme entre le taux de change réel et ses fondamentaux économiques. Le modèle de base s'écrit comme suit :

$$
R_{E E R_{i, t}}=\alpha_{i}+\beta^{\prime} Z_{i, t}+\varepsilon_{i, t}
$$

Dans ce modèle, la variable $R E E R_{i, t}$, représente le taux de change effectif réel du pays $i$ à la période $t$ exprimé au certain; $Z_{i, t}$, est le vecteur des variables explicatives ; $\alpha_{i}$ est une constance spécifique à chaque pays; $\beta$ est le vecteur de paramètres saisissant la relation à long terme entre $R E E R_{i, t}$ et $Z_{i, t}$; et $\varepsilon_{i, t}$ est le terme d'erreur de la régression.

Notons que toutes les autres variables sont exprimées en logarithme.

\subsection{Sources et construction des données}

L'étude se rapporte aux huit (8) pays de l'UEMOA ${ }^{19}$ sur la période 1999 - 2016. Les données sont annuelles et proviennent de plusieurs sources: les bases de données de la Banque Mondiale (WDI), des Nations Unies (UN COMTRADE) et de la CNUCED.

\section{La variable dépendante}

Le taux de change effectif réel (REER) est défini par le prix des biens échangés par rapport au prix des biens (domestiques) non échangés ${ }^{20}$.

\footnotetext{
${ }^{19}$ Bénin, Burkina Faso, Côte d'Ivoire, Guinée-Bissau, Mali, Niger, Sénégal et Togo

${ }^{20}$ Il existe deux définitions du taux de change effectif réel. La première définition appelée taux de change réel interne est la plus appropriée et la plus utilisée dans la littérature concernant les pays en développement. Cependant sa mesure pose un problème, les indices
} 
On a :

$$
R E E R_{i}=N E E R \times \prod_{j=1}^{n}\left(\frac{p_{i}}{p_{j}}\right)^{\alpha_{i j}}
$$

Où NEER est le taux de change effectif nominal, $p_{i}$ étant les prix des biens domestiques et $P_{j}$ l'indice des prix des biens échangeables.

Cette variable est fournie par la Base de données de la CNUCED pour la Côte d'Ivoire, la Guinée-Bissau, le Mali, le Niger et le Sénégal ; World Development Indicators (WDI) pour le Togo; les données du Bénin étant manquantes de 1999 à 2003, nous les avons complétées en faisant l'hypothèse que les taux de change effectifs réels évoluent au rythme de $(-1,829 \%)$ par an, soit le taux de croissance moyen des taux de change effectifs réels de ce pays sur la période 2004 - 2016 provenant des données de la CNUCED.

\section{Les variables explicatives}

A long terme, les facteurs affectant la demande de biens non échangeables par rapport aux biens échangeables auront un impact sur le taux de change réel [Edwards Sebastian (1989), Mac Donald (1997), Clark Peter et MacDonald Ronald (1998), Baffes et al. (1999)]. Les fondamentaux et leur impact théorique sont présentés.

- Les termes de l'échange (TDE) sont définis par les prix relatifs des matières premières par rapport à ceux des produits manufacturés (MUV). Selon la méthode de Cashin et al. (2004) et reprise par Coudert et al. (2010), nous avons tenu compte des trois principaux produits d'exportation de chaque pays (voir tableau 2 en annexe). C'est la variable d'intérêt. On peut écrire les termes de l'échange des prix des matières premières comme suit :

$$
T D E=\sum_{k=1}^{3} \alpha_{i}^{k} P_{t}^{K} / M U V_{t}
$$

Avec $\alpha_{i}^{k}$ le poids de la marchandise $k$ dans les exportations du pays

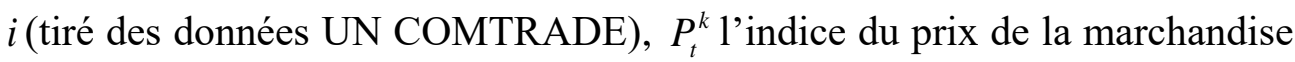
$k$ à la période $t$ (fournis par la CNUCED) et $M U V$ l'indice de la valeur unitaires des exportations des produits manufacturés des pays développés (tiré de la base des données de la CNUCED). Les parts des exportations évoluant avec le temps, le poids de chaque produit est la moyenne arithmétique sur la

de prix des biens échangeables et des biens non échangeables nécessaires pour le calculer n'étant pas disponibles, la plupart des auteurs utilisent le Taux de Change Réel interne théorique à savoir le Taux de Change Réel externe. En effet, le Taux de Change Réel externe requiert des séries sur les indices de prix à la consommation, dont disposent tous les pays (Hélène DJOUFELKI, 2005). 
période 2011 - 2017 (voir le tableau 2 en annexe). Selon la théorie, l'augmentation des prix des matières fait prévaloir l'effet revenu sur l'effet de substitution des termes de l'échange d'où une appréciation des taux de change effectif réels.

Par ailleurs, un fait frappant de l'évolution des principaux produits d'exportations de l'UEMOA au cours de ces dernières années (soit de 2001 à 2016) est l'importance de la part des matières énergétiques et minières. Ainsi, le pétrole occupe la première et la deuxième place des exportations, respectivement au Sénégal et en Côte d' Ivoire. De même, l'or est principalement un produit d'exportation au Mali et au Burkina Faso. Logiquement, l'Uranium est le premier produit d'exportation du Niger. Cela semble remettre en cause la spécialisation des pays de l'UEMOA dans l'exportation de matières premières essentiellement agricoles.

- La productivité relative (PROD) définie par le PIB par tête des pays de l'UEMOA relativement aux dix partenaires commerciaux ${ }^{21}$ représente l'effet Balassa-Samuelson. Selon cet effet, un pays où la productivité des biens échangeables par rapport aux biens non échangeables augmente par rapport à celle de ses partenaires commerciaux, verra sa richesse augmentée, ce qui est associé à une augmentation de la demande des biens domestique, et donc une appréciation du taux de change effectif réel. On a :

$$
\text { PROD }_{i t}=\frac{P I B_{i t}}{\prod_{j=1}^{10} P I B_{j t}{ }^{\left(w_{j}\right)}}
$$

Où $P I B_{i}$ est le PIB par habitant du pays $i, P I B_{j}$ est le PIB du partenaire $j$ et le poids de chaque partenaire $w_{j}$ correspond à la moyenne de la part du commerce (exportation) sur la période $2001-2016$ du pays $i$ avec le partenaire $j$. Les données proviennent de la CNUCED.

- Dans la plupart des études empiriques ayant trait aux pays de la zone $\mathrm{CFA}^{22}$, l'aide publique au développement (APD) rapporté au PIB est soumise à l'hypothèse de la «maladie hollandaise » selon laquelle elle

\footnotetext{
${ }^{21}$ Les principaux clients des pays de l'UEMOA sont présentés dans le tableau (Selon les statistiques de UN COMTRADE, en annexe).

${ }^{22}$ La zone CFA est composée de quatorze pays d'Afrique subsaharienne et repartis entre deux zones monétaires. Ce sont huit pays d'Afrique de l'Ouest (Bénin, Burkina Faso, Côte-d'Ivoire, Guinée Bissau, Mali, Niger, Sénégal et Togo) réunis dans l'UEMOA où circule le franc de la Communauté Financière africaine émis par la BCEAO et six pays d'Afrique centrale à savoir le Cameroun, la Centrafrique, le Congo, le Gabon, la Guinée Equatoriale et le Tchad intégrés dans la CEMAC qui utilisent le Franc de Coopération Financière d'Afrique Centrale émis par la BEAC.
} 
est associée à une appréciation des taux de change effectifs réels. Les données viennent de la CNUCED.

- L'effet de la dépense publique (DPU) rapporté au PIB sur le taux de change réel dépendra de sa composition. Si la consommation est dominée par les biens non échangeables (la consommation de biens échangeables), alors le taux de change réel tendrait à s'apprécier (à se déprécier). Autrement dit, l'effet de la consommation publique sur le taux de change réel est ambigu (Ouattara et Strobl, 2008). Nous la tirons de la base de données de la CNUCED.

- L'investissement public (INVEST) sur le PIB est l'un des importants indicateurs utilisés pour évaluer la variabilité du taux de change réel dans les pays de l'UEMOA. Dans la plupart des études, il est considéré comme un facteur de dépréciation du taux de change réel en raison de l'importance des biens d'équipement pour survenir aux besoins d'investissement des pays en développement. Nous l'avons extrait de la base données de la CNUCED.

- L'ouverture économique (OUV) représente ici la politique commerciale et la dépendance des Etats-membres vis-à-vis de l'extérieur. En effet, les régimes de change fixe sont associés à une forte ouverture économique qui s'accompagne d'une dépréciation des taux de change réels. Elle est calculée à l'aide des données de CNUCED; il s'agit de la somme des exportations et importations sur le PIB.

- Nous incluons aussi l'évolution de l'euro par rapport au dollar (EURO) pour apprécier l'impact de la fluctuation de la monnaie d'ancrage sur le taux de change effectif réel. Cette variable est assimilée à la variation nominale du F CFA. Toutes choses égales par ailleurs, une appréciation nominale sera associée à une appréciation réelle. Les données proviennent de la CNUCED

\section{Méthodologie d'estimation}

Nous estimons les liens entre les termes de l'échange des prix de matières premières et le taux de change effectif réel des pays de l'UEMOA en prenant appui sur l'estimateur Pooled Mean Group (PMG) de Pesaran et al., (1999). Avant de procéder aux estimations, nous présentons d'abord les tests préliminaires de stationnarité et de cointégration sur les données de panel des pays de l'UEMOA.

\subsection{Techniques d'intégration et de cointégration en données de panel}

L'approche économétrique du modèle (12) repose sur l'utilisation des techniques d'intégration sur données de panel proposées par Levin, Lin et 
Chu (2002) et Im et al. (2003) ainsi que sur les récents tests de cointégration sur données de panel développés par Westerlund (2007).

\section{Test stationnarité}

Les résultats du test de stationnarité ${ }^{23}$ calculés au moyen du logiciel STAT 14 sont présentés dans le tableau 1 ci-dessous. Seules les variables ln_APD et ln_PROD qui sont stationnaires à niveau. Cependant, toutes les variables sont intégrées en différence première $\mathrm{I}(1)$.

Tableau 1 : Les résultats du test LLC (2002) et IPS (2003)

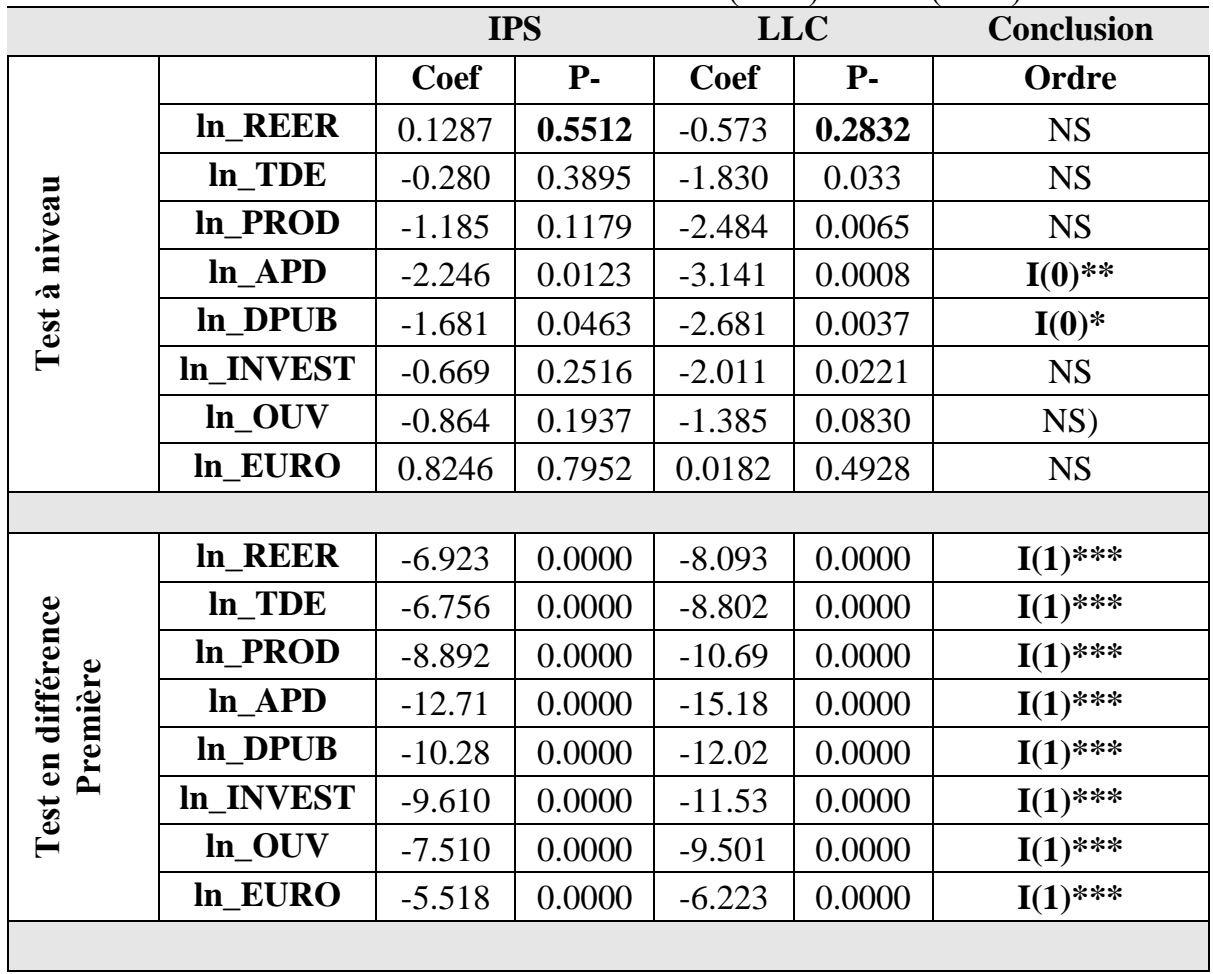

Source : Les auteurs, Notes : stationnaire à $* 10 \%, * * 5 \%, * * * 1 \%$.

Ce résultat inspire plusieurs commentaires. En effet, il ressort de ce tableau que les variables de notre modèle ont conservé une distribution constante dans le temps. Ce résultat consolide notre volonté de concentrer notre étude sur la zone UEMOA dans la mesure où le regroupement des pays

${ }^{23}$ La première étape du processus d'estimation consiste à examiner les propriétés des séries (stationnarité et ordre d'intégration) temporelles de nos données. A ce niveau, les développements récents de la littérature suggèrent que les tests de racine unitaire sur données de panel sont plus puissants que les tests sur séries chronologiques individuelles. Les tests développés récemment sont celui de Breitung (2000), le test de Hadri (2000), le test de Levin et Lin (2002) et le test d'Im Pesaran et Shin (2003). Nous utiliserons dans le cadre de cette étude les deux derniers tests. 
selon un critère géographique accorde un plus grand degré d'homogénéité pour aboutir à des résultats robustes. Par ailleurs, la période post introduction de l'euro a permis de préserver l'homogénéité temporelle et structurelle de notre étude.

\section{Test de cointégration}

Westerlund (2007) a développé quatre nouveaux tests de cointégration qui sont basés, non pas sur les résidus mais sur la dynamique structurelle des relations et qui par conséquent n'imposent aucune restriction sur les facteurs communs.

L'idée est de tester l'hypothèse nulle de non cointégration en vérifiant si le terme de correction d'erreur dans le modèle à correction d'erreur construit à cet effet est significativement égal à zéro. Les quatre tests sont normalement distribués et accommodent assez bien les dynamiques individuelles de courte période, les tendances, les paramètres spécifiques aux individus et les dépendances intra individus. Les deux derniers tests ( $\mathrm{Pt}$ et $\mathrm{Pa}$ ) permettent de tester l'hypothèse nulle d'absence de cointégration contre l'hypothèse alternative selon laquelle le panel dans son ensemble est cointégré alors que les deux premiers tests (Gt et $\mathrm{Ga}$ ) testent l'alternative d'existence d'au moins un individu pour lequel les variables sont cointégrées. Les résultats des tests sont consignés dans le tableau 2.

Tableau 2 : Tests de cointégration (Westerlund, 2007)

\begin{tabular}{|c|c|c|c|c|c|c|c|c|}
\hline \multirow[b]{2}{*}{ Statistics } & \multicolumn{2}{|c|}{ In_reer / In_tde } & \multicolumn{2}{|c|}{ ln_reer / ln_prod } & \multicolumn{2}{|c|}{ In_reer /ln_apd } & \multicolumn{2}{|c|}{ In_reer /ln_dpub } \\
\hline & Coef & P-value & Coef & P-value & Coef & $\begin{array}{c}\text { P- } \\
\text { value }\end{array}$ & Coef & P-value \\
\hline Gt & -3.909 & 0.000 & -3.620 & 0.000 & -6.640 & 0.000 & -4.407 & 0.000 \\
\hline $\mathbf{G a}$ & -12.016 & 0.480 & -14.783 & 0.110 & -15.289 & 0.075 & -7.807 & 0.959 \\
\hline $\mathbf{P t}$ & -7.793 & 0.017 & -7.390 & 0.050 & -7.933 & 0.011 & -9.513 & 0.000 \\
\hline $\mathbf{P a}$ & -13.939 & 0.009 & -12.345 & 0.054 & -13.675 & 0.013 & -14.598 & 0.004 \\
\hline \multirow[b]{2}{*}{ Statistics } & \multicolumn{2}{|c|}{ In_reer / ln_Invest } & \multicolumn{2}{|c|}{ In_reer / ln_ouv } & \multicolumn{4}{|c|}{ In_reer/ln_euro } \\
\hline & Coef & P-value & Coef & P-value & \multicolumn{2}{|c|}{ Coef } & \multicolumn{2}{|c|}{ P-value } \\
\hline Gt & -5.376 & 0.000 & -4.445 & 0.000 & \multicolumn{2}{|c|}{-5.834} & \multicolumn{2}{|c|}{0.000} \\
\hline Ga & -19.212 & 0.001 & -14.744 & 0.113 & \multicolumn{2}{|c|}{-23.798} & \multicolumn{2}{|c|}{0.000} \\
\hline $\mathbf{P t}$ & -12.503 & 0.000 & -6.498 & 0.272 & \multicolumn{2}{|c|}{-15.661} & \multicolumn{2}{|c|}{0.000} \\
\hline $\mathbf{P a}$ & -21.380 & 0.000 & -11.974 & 0.076 & \multicolumn{2}{|c|}{-25.110} & \multicolumn{2}{|c|}{0.000} \\
\hline
\end{tabular}

Source: Les auteurs à partir du logiciel stata 14

Il ressort de ce tableau que l'hypothèse de non cointégration $\left(\mathrm{H}_{0}\right)$ peut être rejetée au seuil de $5 \%$ pour la plupart des variables (selon les statistiques $\mathrm{Pt}$ et $\mathrm{Pa}$ ). Il existe en effet un mécanisme à correction d'erreur. A long terme, les séries ont des évolutions similaires car les déséquilibres de 
court terme entre les variables se compensent dans le temps. En outre, le modèle à correction d'erreur peut être adopté pour le panel tout entier et même pour certains pays pris individuellement ( $\mathrm{Gt}$ et $\mathrm{Ga}$ ).

On peut donc dire à la lumière de ce tableau qu'il existe bien une relation de long terme entre le taux de change effectif réel et l'évolution des prix des matières premières. L'estimation du modèle par le Pooling Mean Group (PMG) nous permettra de confirmer ce résultat.

\subsection{Estimations du modèle}

Le Pooled Mean Group (PMG) de Pesaran et al., (1999) permet d'évaluer la dynamique d'ajustement et le degré de convergence du taux de change effectif réel du F CFA ouest africain vers sa valeur d'équilibre, ce qui justifie l'utilisation de ce Modèle à Correction d'Erreur (MCE) en panel. Cette approche a l'avantage de prendre en compte l'hétérogénéité des paramètres de court terme tout en conservant une homogénéité des paramètres de long terme. En effet, la méthode $\mathrm{PMG}$ se présente comme un modèle autorégressif à retards échelonnés ARDL ${ }^{24}$ comme suit:

$$
R E E R_{i t}=\sum_{j=1}^{p} \lambda_{i t} R E E R_{i, t-j}+\sum_{j=0}^{q} \delta_{i j}^{\prime} Z_{i, t-j}+\mu_{i}+\varepsilon_{i t}
$$

Où $\mu_{i}$ représente les effets fixes individuels, les $\lambda_{i t}$ sont des coefficients affectés aux variables dépendantes individuelles retardées $R E E R_{i, t-j}$, et $\delta_{i j}^{\prime}$ est une matrice de scalaires de format $(1 \times K)$. Si les variables sont cointegrées, alors le terme d'erreur $\varepsilon_{i t}$ est un processus stationnaire. Dans ce cas, le modèle peut prendre la forme d'un modèle à correction d'erreur qui permet de distinguer les dynamiques de court et de long terme.

$$
\Delta R E E R_{i t}=\phi_{i}\left(\operatorname{REER}_{i, t-1}-\beta_{i}^{\prime} Z_{i t}\right)+\sum_{j=1}^{p-1} \lambda^{*}{ }_{i t} \Delta R E E R_{i, t-j}+\sum_{j=0}^{q-1} \delta_{i j}^{* \prime} \Delta Z_{i, t-j}+\mu_{i}+\varepsilon_{i t}
$$

Où $\beta_{i}$ est le vecteur des coefficients de long terme et $\Delta$ l'opérateur de variation entre deux dates successives. Le coefficient d'ajustement, $\phi_{i}$, ainsi que les coefficients de long-terme, $\beta_{i}$ constituent les paramètres d'intérêt. On s'attend à ce que $\phi_{i}<0$. L'un des avantages des modèles ARDL est que les multiplicateurs de court et long termes sont estimés conjointement. En outre, ces modèles autorisent la présence de variables pouvant être intégrées de différents Ordres, soit I(0) et I(1), ou cointégrées (Pesaran et Shin, 1999). Par ailleurs, l'estimateur PMG autorise les coefficients de court terme 
et le coefficient d'ajustement à varier suivant les pays, mais les coefficients de long terme sont identiques pour tous les pays $\left(\beta_{i}=\beta\right)$.

\subsection{Résultats économétriques et interprétations économiques}

Le tableau (3) présente les résultats économétriques. Au regard de la littérature dans la zone CFA (Ouattara et Strobl, 2008 ; Couharde et al, 2011 ; Gnimassoun, 2012), nous avons d'abord retenu trois fondamentaux du taux de change effectif (les termes d'échange des matières premières, l'effet BalassaSamuelson et l'investissement) pour estimer le taux de change effectif réel. Ensuite, nous avons progressivement intégré les autres fondamentaux comme variables de contrôle afin de vérifier la significativité de la variable d'intérêt consécutive à ces ajouts. La dernière régression a abouti à la prise en compte de la monnaie d'ancrage (l'Euro). Conformément à l'estimateur $P M G$, le tableau comporte cinq colonnes présentant chacune une dynamique à long terme au-dessus et une dynamique à court terme au-dessous.

A long terme, l'ensemble des résultats est conforme à nos attentes. Il apparait que les termes de l'échange, l'effet Balassa-Samuelson et l'aide publique présentent des coefficients conduisant à l'appréciation du taux de change effectif réel. Il ressort des colonnes (1) - (4) que seules les deux premières variables sont significatives. En effet, une augmentation de $1 \%$ des prix des principaux produits d'exportation se traduit par une appréciation du taux de change effectif réel de l'ordre de 0,127 à $0,11 \%$ avec une significativité de $5 \%$. Ce résultat est conforme aux conclusions de Cashin et al. (2004) de Coudert al. (2010) et Davin (2014) selon lesquels les termes de l'échange des matières premières sont la variable la plus importante de l'appréciation réelle du taux de change des pays exportateurs de produits de base. Ainsi, il est possible de soutenir que l'augmentation des prix des matières premières dans la zone UEMOA s'accompagne d'une hausse des revenus des ménages, toutes choses égales par ailleurs, d'une augmentation de la consommation des biens domestiques. En ce qui concerne la productivité relative, les résultats confirment les effets d'une hausse des prix des matières et mettent en évidence de l'effet Balassa (1964), selon lequel la hausse du revenu dans la zone UEMOA est associée à une augmentation de la consommation des biens domestiques. Effet, les coefficients du LPRO sont compris entre 0,41 et 0,50 . Les élasticités de cette variable sont aussi plausibles au regard de la littérature dans les pays CFA (Couharde et al, 2011, Ouattara et Strobl, 2008). Dans la colonne (5), nous incluons la variable de l'ancrage monétaire (Euro). Il ressort de cette dernière colonne que l'effet des fluctuations de la monnaie d'ancrage sur le taux de change est, certes, positif mais n'apparait pas significatif. Ce résultat est plausible par rapport aux études de Couharde et al, (2011) ; Gnansounou et Verdier-Chouchane (2012) et Abbé (2017). Selon ces études, les effets de l'appréciation de la monnaie d'ancrage 
sur les comportements de consommations de ménages s'estomperaient au fil des années. Par ailleurs, les effets de cette appréciation sont diversement ressentis par les différents pays de la zone. Cela semble expliquer leur conclusion selon laquelle le risque d'une nouvelle dévaluation dans la zone CFA n'est pas imminent. Cette conclusion est, par ailleurs, confirmé par le terme d'erreur significatif qui est compris entre 0,28 et 0,30 proche de celui des travaux de Couharde et al, (2011) qui est de 0,26 dans la zone CFA. En d'autres termes, le temps de retour à la valeur d'équilibre après un choc du taux de change réel est rapide. Il est de l'ordre de 2 et 3 ans.

Les résultats de la dynamique à court terme sont aussi riches d'enseignement. Il ressort du tableau que les termes de l'échange des matières premières ne sont pas significatifs dans aucune des colonnes. Une explication possible est que les transferts de mains d'œuvre d'une activité suite à une hausse (baisse) brusque des prix des matières ne sont pas immédiats en raison de la spécialisation dans les cultures rentes de la zone UEMOA. De plus, la région UEMOA est reconnue pour produire des matières premières héritées de la période coloniale. En ce qui concerne l'investissement, l'aide publique au développement, l'ouverture économique, les résultats indiquent que ces variables ne permettent pas d'expliquer la fluctuation du taux de change effectif à court terme, comme c'est fréquemment le cas dans la littérature de la zone CFA (Coudert et al. 2011 ; Gnimassoun, 2012). En revanche, la productivité relative et la dépense publique contribuent à apprécier le taux de change effectif réel dans la plupart des régressions. Quant à la monnaie d'ancrage, contrairement, à la dynamique de long terme, elle apprécie significativement le taux de change de change effectif réel à court terme. En effet, une appréciation nominale de $10 \%$ est associée à une appréciation réelle de 3,12\%. Cette forte significativité (1\%) permet d'expliquer les perpétuelles rumeurs de dévaluation suite aux surévaluations subites de l'euro par rapport au dollar états-unien, notamment celles constatées entre 2002 et 2010. Il est donc nécessaire que les autorités monétaires prennent au plus tôt des mesures préventives pour contenir des effets pervers des éventuelles fluctuations brusques de la monnaie d'ancrage par rapport au dollar.

Tableau 3 : Estimation du taux de change réel d'équilibre

\begin{tabular}{cccccc}
\hline & $(1)$ & $(2)$ & $(3)$ & $(4)$ & $(5)$ \\
\hline $\begin{array}{c}\text { Long terme } \\
\text { ln_tde }\end{array}$ & $0.114^{* *}$ & $0.106^{* *}$ & $0.108^{* *}$ & $0.127^{* *}$ & $0.0904^{*}$ \\
& $(2.16)$ & $(2.13)$ & $(2.08)$ & $(2.28)$ & $(1.72)$ \\
ln_prod & & & & & \\
& $0.503^{* *}$ & $0.452^{* *}$ & $0.453^{* *}$ & $0.412^{*}$ & $0.443^{* * *}$ \\
& $(2.43)$ & $(2.31)$ & $(2.19)$ & $(1.95)$ & $(2.80)$ \\
ln_invest & -0.119 & -0.102 & -0.101 & -0.0571 & 0.000228 \\
& $(-1.48)$ & $(-1.37)$ & $(-1.30)$ & $(-0.66)$ & $(0.00)$
\end{tabular}




\begin{tabular}{|c|c|c|c|c|c|}
\hline ln_apd & & $\begin{array}{c}0.0631 \\
(1.56)\end{array}$ & $\begin{array}{c}0.0661 \\
(1.55)\end{array}$ & $\begin{array}{c}0.0583 \\
(1.33)\end{array}$ & $\begin{array}{c}0.0558^{*} \\
(1.72)\end{array}$ \\
\hline ln_dpub & & & $\begin{array}{c}-0.0602 \\
(-0.40)\end{array}$ & $\begin{array}{c}-0.0581 \\
(-0.37)\end{array}$ & $\begin{array}{l}0.133 \\
(1.10)\end{array}$ \\
\hline ln_ouv & & & & $\begin{array}{l}-0.158 \\
(-1.15)\end{array}$ & $\begin{array}{c}-0.0938 \\
(-0.95)\end{array}$ \\
\hline ln_euro & & & & & $\begin{array}{l}0.161 \\
(1.23)\end{array}$ \\
\hline $\begin{array}{c}\text { Court terme } \\
\text { _ec }\end{array}$ & $\begin{array}{c}-0.279^{* * *} \\
(-4.49)\end{array}$ & $\begin{array}{c}-0.295^{* * *} \\
(-4.70)\end{array}$ & $\begin{array}{c}-0.286^{* * *} \\
(-4.41)\end{array}$ & $\begin{array}{c}-0.280^{* * *} \\
(-4.31)\end{array}$ & $\begin{array}{c}-0.302^{* * *} \\
(-4.82)\end{array}$ \\
\hline dln_tde & $\begin{array}{c}0.0160 \\
(0.79)\end{array}$ & $\begin{array}{c}0.0117 \\
(0.58)\end{array}$ & $\begin{array}{c}0.00998 \\
(0.49)\end{array}$ & $\begin{array}{c}0.00782 \\
(0.38)\end{array}$ & $\begin{array}{c}-0.0183 \\
(-1.07)\end{array}$ \\
\hline dln_prod & $\begin{array}{c}0.287^{* *} \\
(2.19)\end{array}$ & $\begin{array}{c}0.257^{* *} \\
(1.97)\end{array}$ & $\begin{array}{l}0.234^{*} \\
(1.75)\end{array}$ & $\begin{array}{l}0.235^{*} \\
(1.75)\end{array}$ & $\begin{array}{l}0.124 \\
(1.16)\end{array}$ \\
\hline dln_invest & $\begin{array}{c}-0.0470 \\
(-1.56)\end{array}$ & $\begin{array}{c}-0.0573^{*} \\
(-1.90)\end{array}$ & $\begin{array}{c}-0.0532^{*} \\
(-1.74)\end{array}$ & $\begin{array}{c}-0.0590^{*} \\
(-1.79)\end{array}$ & $\begin{array}{c}-0.0318 \\
(-1.21)\end{array}$ \\
\hline dln_apd & & $\begin{array}{c}0.00705 \\
(0.58)\end{array}$ & $\begin{array}{c}0.00603 \\
(0.49)\end{array}$ & $\begin{array}{c}0.00780 \\
(0.63)\end{array}$ & $\begin{array}{c}0.00946 \\
(0.96)\end{array}$ \\
\hline dln_dpub & & & $\begin{array}{c}-0.0320 \\
(-0.60)\end{array}$ & $\begin{array}{c}-0.0352 \\
(-0.66)\end{array}$ & $\begin{array}{c}-0.0798^{*} \\
(-1.88)\end{array}$ \\
\hline dln_ouv & & & & $\begin{array}{c}0.0157 \\
(0.29)\end{array}$ & $\begin{array}{c}-0.0108 \\
(-0.26)\end{array}$ \\
\hline dln_euro & & & & & $\begin{array}{c}0.312^{* * *} \\
(6.16)\end{array}$ \\
\hline _cons & $\begin{array}{c}1.488^{* * *} \\
(4.75) \\
\end{array}$ & $\begin{array}{c}1.599^{* * *} \\
(5.02)\end{array}$ & $\begin{array}{c}1.517^{* * * *} \\
(4.52)\end{array}$ & $\begin{array}{c}1.451^{* * *} \\
(4.26)\end{array}$ & $\begin{array}{c}1.734^{* * *} \\
(5.06)\end{array}$ \\
\hline
\end{tabular}

Source : Estimation économétrique des auteurs

\section{Conclusion et recommandations}

Cette étude avait pour objectif d'évaluer les effets des fluctuations des prix des matières premières sur les taux de change effectif réels des huit (8) pays de l'UEMOA sur la période 1999 -2016. A cette fin, nous nous sommes basés sur un modèle théorique de Cashin et al, (2004) et de l'approche comportementale BEER. Par la suite, nous avons eu recours aux techniques d'intégration, de cointégration et d'un estimateur PMG (Pooled Mean Group) de Pesaran et al, (1999) pour évaluer les dynamiques de court et 
de long terme ainsi que le degré de convergence du taux de change effectif réel du F CFA ouest africain vers sa valeur d'équilibre.

Nous montrons que, à long terme, les termes l'échange, l'effet Balassa-Samuelson et l'aide publique présentent des coefficients scientifiques conduisant à l'appréciation du taux de change effectif réel. Il apparait de nos résultats que les termes de l'échange des matières, définis par leurs valeurs relatives par rapport à celles des produits manufacturés, sont le principal facteur du taux de change réel à long terme. Cependant, à court terme, les termes de l'échange des matières premières ne sont pas significatifs. Inversement, la monnaie d'ancrage impacte la dynamique de court terme du taux de change réel, mais n'aide pas à expliquer la dynamique de long terme. Ces principaux résultats appellent plusieurs enseignements. D'abord, il est possible de soutenir que l'augmentation des prix des matières premières dans la zone UEMOA s'accompagne d'une hausse des revenus des ménages dans l'UEMOA, toutes choses égales par ailleurs, d'une augmentation de la consommation des biens domestiques. Par contre, ce comportement s'observe uniquement qu'à long terme. En effet, à court terme, les transferts de mains d'œuvre d'une activité suite à une hausse (baisse) brusque des prix des matières ne sont pas immédiats en raison de la spécialisation de l'UEMOA dans les cultures rentes. C'est donc le lieu d'inviter les autorités à adopter des politiques agricoles et d'exportations à long terme. Le second enseignement est que les effets de l'appréciation de la monnaie d'ancrage sur les comportements de consommation de ménages s'estompent rapidement. Par conséquent, le risque d'une nouvelle dévaluation dans la zone CFA n'est pas imminent. Toutefois, les fluctuations brutes ont tendance à créer des situations de panique dans la population. C'est pourquoi, il s'avère nécessaire que les autorités monétaires prennent au plus tôt des mesures préventives pour contenir des effets pervers des éventuelles fluctuations brusques de la monnaie d'ancrage par rapport au dollar.

\section{References:}

1. Baffes, J.N., Elbadawi I. A., O'Connell, \& Stephen A. (1997), "Singleequation estimation of the equilibrium real exchange rate", Policy Research Working Paper, Series 1800, The World Bank.

2. Bodart V., Candelon B. et Carpantier J-F. (2011), "Real Exchange Rates, Commodity Prices and Structural Factors in Developing Countries", Institut de Recherches Economiques et Sociales, Discussion Paper 2011-45

3. Cashin, P., Céspedes, L.F. and Sahay, R., (2004). "Commodity currencies and the real exchange rate", Journal of Development Economics, 75(1), pp.239-268. 
4. Chen, Y.-C. and K. Rogoff (2003). "Commodity currencies", Journal of international Economics 60(1): 133-160.

5. Clark, P. \&MacDonald R (1997), ''Exchange rates and economic fundamentals: a methodological comparison of BEERs and FEERs', IMF Working Paper 98/00, International Monetary Fund.

6. Coudert, V., Couharde, C. and Mignon, V., (2010). "Taux de change des pays exportateurs de matières premières. L'importance des termes de l'échange et de la monnaie d'ancrage', Revue économique 2010/3 (Vol. 61), p. 499-509.

7. Coudert, V., Couharde, C. and Mignon, V., (2015). "On the impact of volatility on the real exchange rate-terms of trade nexus: Revisiting commodity currencies". Journal of International Money and Finance, 58, pp.110-127.

8. Dauvin, M., (2014). "Energy prices and the real exchange rate of commodity-exporting countries." International Economics 137: 5272.

9. De Gregorio J., Wolf H.C. (1994), « Terms of trade, productivity and the real exchange rate », nber Working Paper 4807, Cambridge (Mass.).

10. Edwards, S. (1988), "Exchange rate misalignment in developing countries', World Bank occasional papers, new series.

11. Gnansounou, S. U \&Verdier-Chouchane, A. (2012), “Mésalignement du taux de change effectif réel : Quand faudra-t-il de nouveau dévaluer le franc CFA ?' , Working Papers Series $N^{\circ} 166$, African Development Bank, Tunis, Tunisia.

12. Gnimassoun, B. (2012), “ Mésalignements du franc CFA et influence de la monnaie d'ancrage', Economie et Prévision 2012/2 ( $\mathrm{n}^{\circ} 200-$ 201), p. 91-119.

13. Im, K.S., M.H. Pesaran \& Y. Shin (2003), "Testing for Unit Roots in Heterogeneous Panels", Journal of Econometrics, 115, pp. 53-74.

14. Kohlscheen E.,. Avalos F. H et Schrimpf A. (2016), "When the Walk is not Random: Commodity Prices and Exchange Rates', Monetary and Economic Department, Working Papers, No 551.

15. MacDonald, R., (1997), "What Determines Real Exchange Rates? The Long and Short of It", IMF Working paper, 97/21, International Monetary Fund.

16. MacDonald, R., (1997), "What Determines Real Exchange Rates? The Long and Short of It", IMF Working paper, 97/21, International Monetary Fund.

17. Maurizio M. H. et Kalamova M. M. (2007), "Are there oil currencies? The real exchange rate of oil exporting countries' European Central Bank, Working paper 
18. Pesaran, Shin et Smith (1999). "Pooled Mean Group Estimation of Dynamic Heterogeneous Panel", Journal of the American Statical Association, Vol. 94, n 446, pp. 621-634.

19. Neary p. (1988), "Determinants of the Equilibrium Real Exchange Rate", American Economic Review, 78 (1), p. 210-215.

20. Ouattara, B. et Strobl, E. (2008), "Foreign Aid Inflows and the Real Exchange Rate in the Cfa Franc Zone", Economie Internationale, CEPII research center, issue 4Q, pages 37-52.

21. Westerlund, J. (2007), "Testing for Error Correction in Panel Data". Oxford Bulletin of Economics and Statistics 69(6): pp.709-748.

\section{ANNEXE}

Tableau A1: Synthèse de revue récente sur les termes de change de matières premières et le taux de change effectif réel sur l'Afrique Subsaharienne

\begin{tabular}{|c|c|c|}
\hline $\begin{array}{l}\text { Auteurs et } \\
\text { année de }\end{array}$ & Pays et période d'étude & Enseignements des études \\
\hline $\begin{array}{l}\text { Emanuel } \\
\text { Kohlscheen, } \\
\text { Fernando H. } \\
\text { Avalos et } \\
\text { Andreas } \\
\text { Schrimpf (2016) }\end{array}$ & $\begin{array}{l}\text { Les auteurs examinent la } \\
\text { relation entre les prix de } \\
\text { l'énergie et le taux de change } \\
\text { effectif réel en Afrique du Sud } \\
\text { en utilisant des données } \\
\text { annuelles pour la période de } \\
1970 \text { à } 2014\end{array}$ & $\begin{array}{l}\text { Conformément à littérature, les termes de l'échange, } \\
\text { l'effet Balassa Samuelson, la position nette des avoirs } \\
\text { à l'étranger, l'ouverture commerciale, l'équilibre fiscal } \\
\text { influencent le taux d'intérêt réel. En utilisant des } \\
\text { techniques et des procédures de test de cointégration } \\
\text { à long terme, les auteurs fournissent des preuves de } \\
\text { l'existence de monnaies énergétiques en Afrique du } \\
\text { Sud. }\end{array}$ \\
\hline $\begin{array}{l}\text { Magali Dauvin } \\
\text { (2014) }\end{array}$ & $\begin{array}{l}\text { L'étude considère deux } \\
\text { groupes de pays : } 10 \\
\text { exportateurs d'énergie et } 23 \\
\text { pays exportateurs de matières } \\
\text { premières, au cours de la } \\
\text { période } 1980-2011 .\end{array}$ & $\begin{array}{l}\text { L'étude montre une relation positive à long terme } \\
\text { entre les termes de l'échange énergétiques et le taux } \\
\text { de change effectif réel des pays exportateurs } \\
\text { d'énergie: une hausse de } 10 \% \text { du prix de l'énergie } \\
\text { entraîne une appréciation de devise. Toutefois, } \\
\text { lorsque le marché du pétrole est calme, les taux de } \\
\text { change effectifs réels des pays exportateurs d'énergie } \\
\text { et de produits de base ne sont pas déterminés par leurs } \\
\text { termes de l'échange mais par d'autres fondamentaux } \\
\text { habituels. }\end{array}$ \\
\hline $\begin{array}{c}\text { BodartV., } \\
\text { Candelon B. and } \\
\text { Carpantier J-F. } \\
\text { (2011) }\end{array}$ & $\begin{array}{l}\text { L'étude porte sur les valeurs } \\
\text { annuelles des exportations de } \\
42 \text { produits pour } 65 \text { pays en } \\
\text { développement et émergents } \\
\text { sur la période } 1988-2007 \text {. }\end{array}$ & $\begin{array}{l}\text { Il existe une relation positive entre les taux de change } \\
\text { réels et les prix des matières premières dans les pays } \\
\text { en développement spécialisés dans l'exportation d'un } \\
\text { produit primaire principal. Par ailleurs, les facteurs } \\
\text { structurels tels que le régime de change, le degré } \\
\text { d'ouverture financière et commerciale, le degré de } \\
\text { concentration des exportations et le type } \\
\text { d'exportations de matières premières influent sur la } \\
\text { solidité du prix des matières premières. }\end{array}$ \\
\hline
\end{tabular}




\begin{tabular}{|c|c|l|}
\hline $\begin{array}{c}\text { Virginie } \\
\text { Coudert, Cécile } \\
\begin{array}{c}\text { Couharde, } \\
\text { Valérie Mignon } \\
(2010),\end{array}\end{array}$ & $\begin{array}{c}\text { Les données sont annuelles et } \\
\text { couvrent la période } 1980 \text { à } \\
\text { 2007. L'étude concerne 68 } 68 \\
\text { pays développé et en } \\
\text { développement dont } 52 \text { pays } \\
\text { exportateurs de matières et 16 } 16 \\
\text { pays exportateurs de pétrole. } \\
\text { taux de change réels, tant pour les pays exportateurs } \\
\text { de matières premières que pour ceux qui exportent du } \\
\text { pétrole. De même, la productivité relative et les actifs } \\
\text { extérieurs nets jouent aussi un rôle dans cette } \\
\text { évolution. } \\
\text { Dans cette étude, des pays hétérogènes à savoir des } \\
\text { pays développés et en voie développement } \\
\text { constituent le panel des exportateurs de matières } \\
\text { premières. }\end{array}$ \\
\hline
\end{tabular}

\section{Source : Les auteurs}

Tableau A 2: Principaux produits d'exportations des pays de l'UEMOA entre 2011 et 2017

\begin{tabular}{|c|c|c|c|c|c|c|}
\hline \multirow{2}{*}{ Pays } & \multicolumn{3}{|c|}{ Principaux produits d'exportations } & \multicolumn{3}{|c|}{$\begin{array}{l}\text { Part du produit dans les } \\
\text { exportations totales en } \%\end{array}$} \\
\hline & 1 & 2 & 3 & 1 & 2 & 3 \\
\hline Bénin & Coton & $\begin{array}{c}\text { Fruits } \\
\text { comestible } \\
\text { (orange) }\end{array}$ & Fer et acier & 45,76 & 9,50 & 6,08 \\
\hline $\begin{array}{l}\text { Burkina } \\
\text { Faso }\end{array}$ & Coton & $\begin{array}{c}\text { Métaux } \\
\text { précieux (or) }\end{array}$ & $\begin{array}{l}\text { Graines et fruits } \\
\text { oléagineux }\end{array}$ & 37,70 & 36,47 & 6,378 \\
\hline $\begin{array}{l}\text { Côte } \\
\text { d'Ivoire }\end{array}$ & Cacao & $\begin{array}{l}\text { Huile de } \\
\text { pétrole }\end{array}$ & Caoutchouc & 35,91 & 22,46 & 5,28 \\
\hline $\begin{array}{l}\text { Guinée } \\
\text { Bissau }\end{array}$ & $\begin{array}{c}\text { Fruits } \\
\text { oléagineux } \\
\text { (d'acajou) }\end{array}$ & $\begin{array}{l}\text { Poissons et } \\
\text { crustacés }\end{array}$ & Bois (grume) & 72,31 & 5,24 & 2,41 \\
\hline Mali & $\begin{array}{l}\text { Métaux } \\
\text { précieux } \\
\text { (Or) }\end{array}$ & Coton & $\begin{array}{l}\text { Animaux vivants } \\
\text { (bœuf) }\end{array}$ & 52,24 & 13,42 & 3,66 \\
\hline Niger & $\begin{array}{l}\text { Minerais } \\
\text { (Uranium) }\end{array}$ & $\begin{array}{l}\text { Animaux } \\
\text { vivants } \\
\text { (bœuf) } \\
\end{array}$ & Coton & 44,76 & 12,53 & 3,26 \\
\hline Sénégal & $\begin{array}{l}\text { Huile de } \\
\text { pétrole }\end{array}$ & $\begin{array}{l}\text { Poissons et } \\
\text { crustacés }\end{array}$ & $\begin{array}{c}\text { Produits chimiques } \\
\text { inorganiques } \\
\text { (acide } \\
\text { phosphorique) }\end{array}$ & 19,25 & 14,43 & 9,82 \\
\hline Togo & Phosphate & Coton & Caoutchouc & 33,94 & 8,62 & 6,78 \\
\hline
\end{tabular}

Source : Base des statistiques de UN COMTRADE (2018) et calculs des auteurs 
Tableau A3 : Principaux partenaires commerciaux de l'UEMOA pour les exportations de 2001 à 2016

\begin{tabular}{|c|c|}
\hline Etats membres & Partenaires commerciaux \\
\hline Bénin & $\begin{array}{c}\text { Chine }(16,50 \%) \text {, Nigéria }(13 \%) \text {, Inde }(11,27 \%) \text {, Indonésie }(4,45 \%) \text {, } \\
\text { Niger }(4,42 \%) \text {, Malaisie }(3,65 \%) \text {, Vietnam }(3,27 \%) \text {, Tchad }(3,22 \%) \text {, } \\
\text { Côte d'Ivoire }(2,50 \%) \text {, Ghana }(2,81 \%) \text {. }\end{array}$ \\
\hline Burkina Faso & $\begin{array}{c}\text { Faso : Togo }(28,44) \text {, Ghana }(13,12 \%) \text {, Suisse }(12,32 \%) \text {, Côte } \\
\text { d'Ivoire }(7,42 \%) \text {, Niger }(3,42 \%) \text {, Singapour }(2,28 \%) \text {, Belgique }(1, \\
98 \%) \text {, Bénin }(1,4 \%) \text {, Italie }(1,32 \%) \text {, Japon }(0,98 \%)\end{array}$ \\
\hline Côte d'Ivoire & $\begin{array}{c}\text { France }(12,13 \%) \text {, Pays-Bas }(12,10 \%) \text {, Etats-Unis }(8,87 \%) \text {, Nigéria } \\
(6 \%) \text {, Allemagne }(4,67 \%) \text {, Ghana }(4,33 \%) \text {, Burkina Faso }(3,51 \%) \text {, } \\
\text { Belgique }(3,16 \%) \text {, Italie }(3,16 \%) \%) \text {, Mali }(3,05 \%) .\end{array}$ \\
\hline Guinée -Bissau & $\begin{array}{c}\text { Inde }(70,4 \%) \text {, Singapour }(25,1 \%) \text {, Portugal }(1,1 \%) \text {, Sénégal }(1,01) \text {, } \\
\text { Chine }(0,54 \%) \text {, Panama }(0,8 \%) \text {, Italie }(0,2 \%) \text {, Espagne }(0,17 \%), \\
\text { Pays-Bas }(0,1) \text {, Corée, RPD }(0,06 \%) .\end{array}$ \\
\hline Mali & $\begin{array}{c}\text { France }(3,23 \%) \text {, Chine }(3,05 \%) \text {, Thaïlande }(1,70 \%) \text {, Burkina Faso } \\
(1,63 \%) \text {, Italie }(1,31 \%) \text {, Vietnam }(1,21 \%) \text {, Malaisie }(1,17 \%), \\
\text { Allemagne }(0,86 \%) \text {, Belgique }(0,79 \%) \text {, Inde }(0,78 \%),\end{array}$ \\
\hline Niger & $\begin{array}{c}\text { Chine }(17,18 \%) \text {, France }(16,11 \%) \text {, Etats-Unis }(7,65 \%) \text {, Côte } \\
\text { d'Ivoire }(6,39 \%) \text {, Nigéria }(5,61 \%) \text {, Japon }(4,07 \%) \text {, Togo }(3,34 \%) \text {, } \\
\text { Inde }(3,04 \%) \text {, Pays-Bas }(2,64 \%) \text {, Bénin }(2,17 \%) \text {. }\end{array}$ \\
\hline Sénégal & $\begin{array}{c}\text { Mali (17,29\%), Suisse }(15,85 \%) \text {, Inde }(10,58 \%) \text {, France }(7,16 \%) \text {, } \\
\text { Gambie }(4,1 \%) \text {, Espagne }(3,50 \%) \text {, Guinée }(3,50 \%) \text {, Mauritanie }(3,06 \\
\text { \%), Guinée-Bissau }(2,78 \%) \text {, (Côte d'Ivoire }(3,29 \%) \text {. }\end{array}$ \\
\hline Togo & $\begin{array}{c}\text { Burkina Fao (13,59\%), Bénin }(12,05 \%) \text {, Ghana }(10,89 \%) \text {, Niger } \\
(7,51 \%) \text {, Inde }(6,36 \%) \text {, Nigéria }(5,23 \%) \text {, Mali }(4,45 \%) \text {, Pays-Bas } \\
(2,81 \%) \text {, France }(1,71 \%) \text {,Chine }(1,61 \%),\end{array}$ \\
\hline
\end{tabular}

Source : Statistiques de UN COMTRADE (2018)

Graphique A1 : Taux de change effectif réel et prix des matières premières dans l’UEMOA

\section{Bénin}

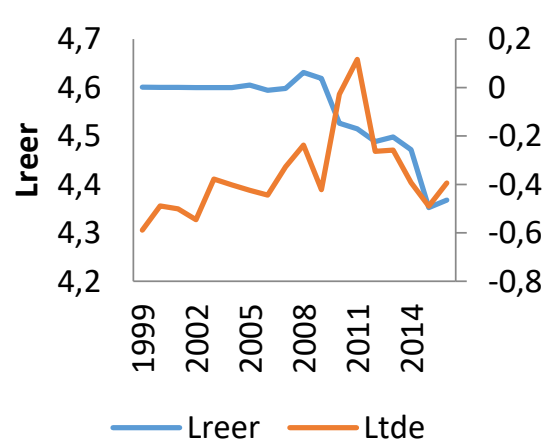

\section{Burkina Faso}

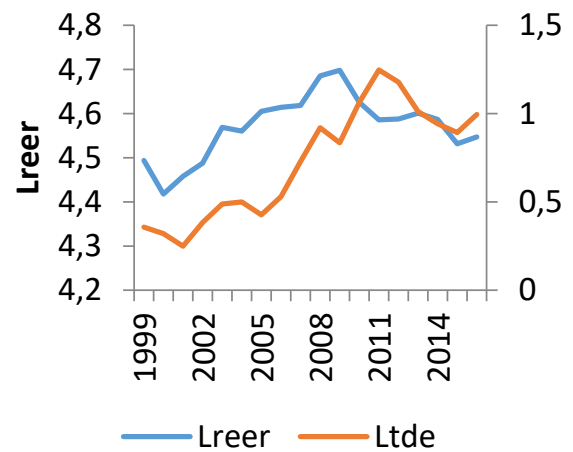




\section{Côte d'Ivoire}

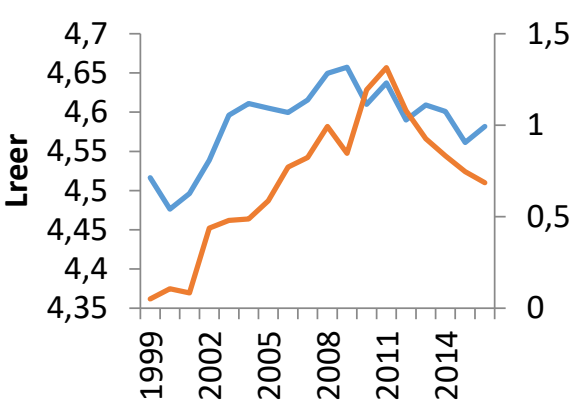

Lreer Ltde

Mali

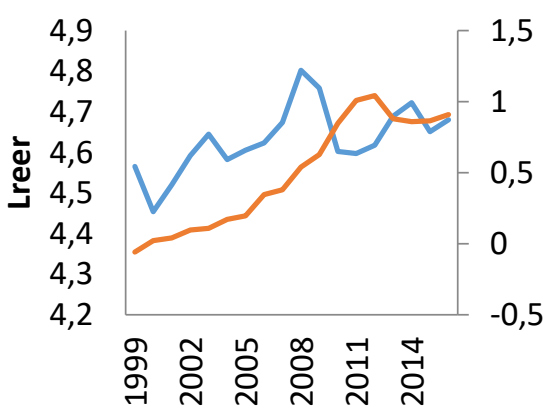

$\longrightarrow$ Lreer Ltde

Sénégal

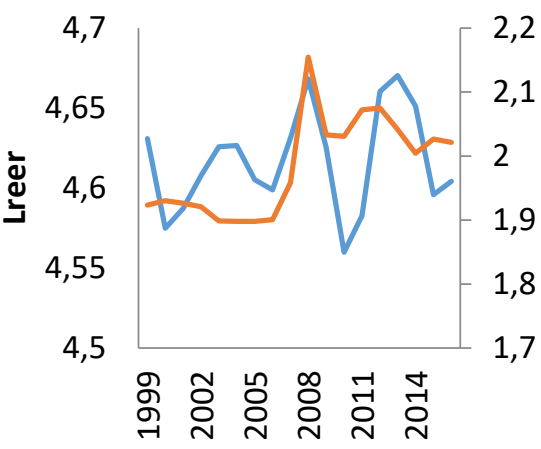

Lreer Ltde

\section{G-Bissau}

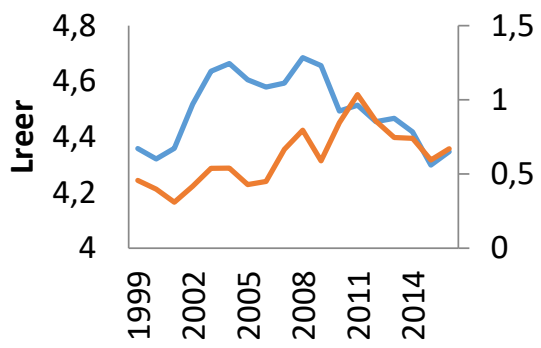

Lreer L Ltde

Niger

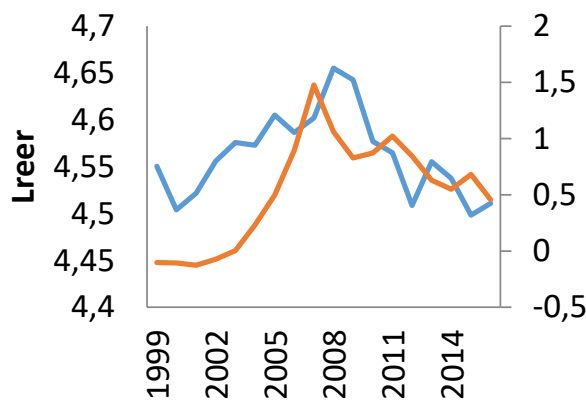

Lreer L Ltde

\section{Togo}

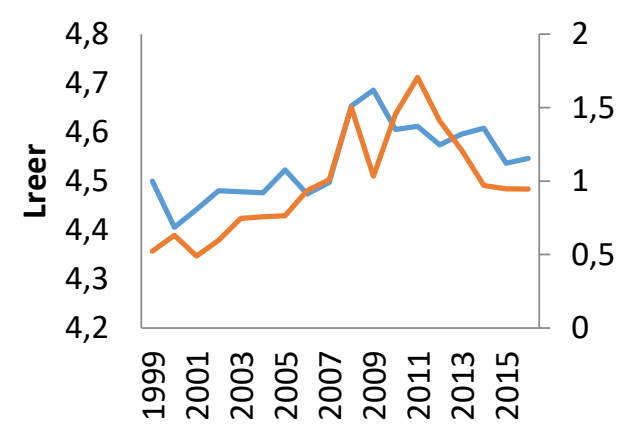

